\title{
A Convergent Reaction-Diffusion Master Equation
}

\author{
Samuel A. Isaacson ${ }^{a}$ ) \\ Department of Mathematics and Statistics \\ Boston University \\ Boston, MA 02215, USA
}

The reaction-diffusion master equation (RDME) is a lattice stochastic reactiondiffusion model that has been used to study spatially distributed cellular processes. The RDME is often interpreted as an approximation to spatially-continuous models in which molecules move by Brownian motion and react by one of several mechanisms when sufficiently close. In the limit that the lattice spacing approaches zero, in two or more dimensions, the RDME has been shown to lose bimolecular reactions. The RDME is therefore not a convergent approximation to any spatially-continuous model that incorporates bimolecular reactions. In this work we derive a new convergent RDME (CRDME) by finite volume discretization of a spatially-continuous stochastic reaction-diffusion model popularized by Doi. We demonstrate the numerical convergence of reaction time statistics associated with the CRDME. For sufficiently large lattice spacings or slow bimolecular reaction rates, we also show the reaction time statistics of the CRDME may be approximated by those from the RDME. The original RDME may therefore be interpreted as an approximation to the CRDME in several asymptotic limits.

a) Electronic mail: isaacson@math.bu.edu 
Computational models of biochemical systems within individual cells have become a common tool used in studying cellular processes and behavior ${ }^{1-4}$. The spatially distributed nature of chemical pathways inside cells may, in certain cases, be more accurately modeled by including the explicit spatial movement of molecules. Examples of such processes include whether signals can propagate from the plasma membrane to nucleus ${ }^{5}$, how cell shape can modify information flow in signaling networks ${ }^{1}$, how the variable density of chromatin influences the search time of proteins for DNA binding $\operatorname{sites}^{6}$, or how different regions of cytosolic space may separate to different chemical states ${ }^{7}$.

One important consideration in developing these models is that at the scale of a single cell many biochemical processes are stochastic ${ }^{8-10}$. Stochastic reaction-diffusion models have been used to account for the stochasticity inherent in the chemical reaction process and the diffusion of proteins and mRNAs. These models approximate individual molecules as points or spheres diffusing within cells. They are more macroscopic descriptions than quantum mechanical or molecular dynamics models, which can resolve detailed interactions between a few molecules on timescales of milliseconds ${ }^{11}$. They are more microscopic descriptions than deterministic three-dimensional reaction-diffusion PDEs for the average concentration of each species of molecule.

Three stochastic reaction-diffusion models that have been used to study cellular processes are: the Doi model ${ }^{12-14}$, the Smoluchowski diffusion limited reaction model ${ }^{15,16}$, and the reaction-diffusion master equation $(\mathrm{RDME})^{17-22}$. In the Doi model ${ }^{13,14}$ positions of molecules are represented as points undergoing Brownian motion. Bimolecular reactions between two molecules occur with a fixed probability per unit time when two reactants are separated by less than some specified "reaction radius". The Smoluchowski model differs by representing bimolecular reactions in one of two ways; either occurring instantaneously (called pure absorption), or with fixed probability per unit time (called partial absorption), when two reactants' separation is exactly the reaction-radius ${ }^{15,16}$. Two possible reactants are not allowed to approach closer than their reaction-radius. A more microscopic version of the Smoluchowski model approximates molecules as spheres, allowing for volume exclusion and collisions between non-reactive molecules. In each of the models unimolecular reactions represent internal processes. They are assumed to occur with exponentially distributed times based on a specified reaction-rate constant.

The RDME can be interpreted as an extension of the non-spatial chemical master equa- 
tion (CME) $)^{17-19,23}$ model for stochastic chemical kinetics. In the RDME space is partitioned by a mesh into a collection of voxels. The diffusion of molecules is modeled as a continuous time random walk on the mesh, with bimolecular reactions occurring with a fixed probability per unit time for molecules within the same voxel. Within each voxel molecules are assumed well-mixed (i.e. uniformly distributed), and molecules of the same species are indistinguishable. Molecules are treated as points in the RDME, and in the absence of chemical reactions the continuous time random walk each molecule undergoes converges to the Brownian motion of a point particle as the mesh spacing approaches zero ${ }^{21,24,25}$. Mathematically, the RDME is the forward Kolmogorov equation for a continuous-time jump Markov process.

In each of the three models the state of the chemical system is given by stochastic processes for the number of molecules of each chemical species and the corresponding positions of each molecule (where in the RDME a molecule's position corresponds to the voxel containing that molecule). There are two different mathematical formulations of each model; either coupled systems of equations for the probability densities of having a given state at a specified time, or equations for the evolution of the stochastic processes themselves. The former description leads to, possibly infinite, coupled systems of partial integral differential equations (PIDEs) for the Doi/Smoluchowski models, and, possibly infinite, coupled systems of ordinary differential equations (ODEs) for the RDME. The high dimensionality of these equations for typical biological systems prevents the use of standard numerical methods for PDEs/ODEs in low-dimensions. Instead, the probability density solutions to these equations are approximated by simulating the underlying stochastic processes with Monte Carlo methods ${ }^{26-33}$.

When the RDME is interpreted as an independent physical model, there is a natural, nonzero, lower bound on the lattice spacing in systems with bimolecular reactions ${ }^{7,21,34}$. In its most basic form, this bound arises from the physical assumption that the timescale for molecules to become well-mixed within a voxel is smaller than that for bimolecular reactions to occur. The use of a well-mixed bimolecular reaction mechanism within each voxel is then physically justified. While the RDME can be used as an independent physical model, in applications it is often interpreted as a formal approximation of the Doi or Smoluchowski models $^{24,35}$. It is important to stress that the RDME can probably be interpreted as a (non-convergent) physical approximation to a large number of distinct, microscopic, spatially-continuous models. For example, Gillespie has developed an argument for the 
physical validity of the RDME with sufficiently large lattice spacings as a (non-convergent) approximation to a model that involves the diffusion of hard-spheres which may react upon collision $^{36}$.

In this work we interpret the RDME as an attempt to approximate to the Doi model. While previous work on improving the accuracy of the RDME as an approximation to microscopic models has focused on the (point-particle) version of the Smoluchowski model ${ }^{34,35}$, we showed in $^{24}$ that the RDME can be seen to approximate a version of the Doi model. A number of studies of microscopic stochastic reaction-diffusion processes have been based on the Doi model ${ }^{26,37-41}$, including recent work modeling the dynamics of intracellular calcium release $^{42}$. As we discuss in the next section, recent studies ${ }^{41,43}$ demonstrate that the Doi model offers comparable accuracy to the point-particle version of the Smoluchowski model.

The assumption that molecules are represented by point particles is consistent with the use of continuous time random walks to approximate molecular diffusion. In systems without bimolecular reactions, the RDME should converge as the lattice spacing approaches zero to a model of point particles that move by Brownian motion and may undergo linear reactions. For systems in which no reactions are allowed, the RDME can be shown to converge to a model for a collection of point-particles that undergo independent Brownian motions ${ }^{24}$. This model is the standard starting point for deriving modified spatial hopping rates in the RDME when incorporating new spatial transport methods ${ }^{6,44,45}$ or other types of lattice discretizations $^{21,25,45,46}$. In the remainder, when we refer to the Smoluchowski model we will assume that molecules are represented by points unless stated otherwise.

In practice, using the RDME to approximate either of the Doi or Smoluchowski models for systems including bimolecular reactions can be problematic. It has been shown that in the continuum limit where the mesh spacing in the RDME is taken to zero bimolecular reactions are lost ${ }^{22,34}$ (in two or more dimensions). That is, the time at which two molecules will react becomes infinite as the mesh spacing is taken to zero. This result is consistent with the physical lower bound on the lattice spacing, and as such the RDME can only provide an approximation to the Doi or Smoluchowski models for mesh spacings that are neither too large or too small ${ }^{47}$. The error in this approximation can not be made arbitrarily small; in ${ }^{47}$ we found that for biologically relevant parameters values, the standard RDME could at best approximate within five to ten percent the binding time distribution for the two-molecule $\mathrm{A}+\mathrm{B} \rightarrow \varnothing$ in the Smoluchowski model $\left(\right.$ in $\left.\mathbb{R}^{3}\right)$. 
In this work we offer one possible method to overcome this lower limit on the approximation error. We discretize the Doi model so as to obtain a forward Kolmogorov equation describing a continuous-time jump Markov process on a lattice similar to the RDME. We seek a discretization of this form so that we may use Monte Carlo methods to simulate the underlying stochastic process for systems of many reacting molecules. The convergent RDME (CRDME) we obtain approximates the Doi model, but retains bimolecular reactions as the mesh spacing approaches zero. This is accomplished by decoupling the region in which two molecules can react from the choice of mesh. Consider the two molecule $\mathrm{A}+\mathrm{B} \rightarrow \varnothing$ reaction. In the RDME, an A molecule within a given voxel can only react with $\mathrm{B}$ molecules within the same voxel. The CRDME enforces that molecules with separation smaller than a specified reaction-radius can react, as in the Doi model. In the CRDME molecules are assumed well-mixed within the voxel containing them, and so their position is known only to the scale of one voxel. An A molecule within a given voxel can then react with any $\mathrm{B}$ molecule in any nearby voxel where the minimal distance between points within the two voxels is less than the reaction radius. In this way, when the lattice spacing is greater than the reaction radius, an $\mathrm{A}$ molecule within a specified voxel can only react with $\mathrm{B}$ molecules in nearest neighbor voxels (including diagonal neighbors). As the lattice spacing is made smaller than the reaction-radius, reactions can occur between molecules that are separated by multiple voxels. The number of voxels separating two voxels for which a reaction can occur increases as the lattice spacing is decreased. The probability per unit time for two molecules to react in the CRDME is a non-increasing function of the separation between the voxels containing the two molecules. It decreases to zero for all pairs of voxels in which the minimal distance between any two points is larger than the reaction radius.

For $T$ the random variable for the time at which two molecules react, we show in twodimensions by numerical simulation that both the survival time distribution, $\operatorname{Pr}[T>t]$, and the mean reaction time converge to finite values as the mesh spacing approaches zero in the CRDME (in contrast to the RDME). We also verify by comparison with Brownian dynamics simulations ${ }^{26,41}$ that both the survival time distribution and mean reaction time converge to that of the Doi model.

The new CRDME retains many of the benefits of the original RDME model, and allows the re-use of the many extensions of the RDME that have been developed. Examples of these extensions include Cartesian-grid methods for complex geometries ${ }^{21}$; the incorporation 
of drift due to potentials ${ }^{6,44}$; advective velocity fields ${ }^{45}$; non-Cartesian meshes $^{25}$; time dependent domains ${ }^{48}$; GPU optimized simulation methods ${ }^{49}$; adaptive mesh refinement techniques (AMR) to improve the approximation of molecular diffusion ${ }^{46}$; and multiscale couplings to more macroscopic models ${ }^{50}$.

In addition to retaining bimolecular reactions as the mesh spacing approaches zero, we show that the CRDME is approximable by the RDME for appropriate mesh spacings and parameter choices. It should be noted that the approach we take in no way invalidates the standard RDME as an approximation to microscopic, spatially-continuous models for sufficiently large lattice spacings. Instead, the new CRDME offers an approximation of the Doi model in which the approximation error can be controlled (through mesh refinement). The finite volume discretization approach we use also suggests a possible method for trying to derive CRDMEs that approximate the Smoluchowski model.

We begin in the next section by reviewing several of the previous approaches that have been used to improve the accuracy of the RDME in approximating spatially continuous particle-based models. In Section II we introduce the general Doi and RDME models for the multiparticle $\mathrm{A}+\mathrm{B} \rightarrow \mathrm{C}$ reaction. These abstract formulations illustrate that all bimolecular reaction terms in the Doi model correspond to identical two-body interactions. We next derive our new CRDME for the two-particle annihilation reaction $\mathrm{A}+\mathrm{B} \rightarrow \varnothing$ in Section III. The numerical convergence of reaction time statistics associated with this CRDME are demonstrated in Section IV. In Section $V$ we show how the RDME may be interpreted as an asymptotic approximation to the CRDME for large mesh sizes or small reaction radii. The CRDME allows molecules in neighboring voxels to react, suggesting the question of where to place newly created reaction products for the $\mathrm{A}+\mathrm{B} \rightarrow \mathrm{C}$ reaction. We assume bimolecular reaction products in the Doi model are placed at the center of mass of the two reactants. In Section VI we derive a general CRDME approximation of the Doi model for where to place a newly created $\mathrm{C}$ molecule following the bimolecular reaction, $\mathrm{A}+\mathrm{B} \rightarrow \mathrm{C}$. Finally, we conclude in Section VII by summarizing how the standard stochastic simulation algorithm (SSA) for generating realizations of the stochastic process described by the RDME should be modified for the CRDME. 


\section{PREVIOUS WORK}

There are several modified RDME models that have been developed to improve the approximation of bimolecular reactions ${ }^{26,34,35}$. To our knowledge, none of these works provide convergent approximations of a spatially-continuous stochastic reaction-diffusion model as the lattice spacing is taken to zero. Instead, they are each designed to more accurately approximate one specific fixed statistic over a range of lattice spacings that are above some critical size. The works of ${ }^{26,34}$ derive modified, lattice spacing dependent reaction rates for two molecules within the same voxel. $\operatorname{In}^{34}$ the reaction rate is modified to try to match the mean association time for the two-molecule $\mathrm{A}+\mathrm{B} \rightarrow \varnothing$ reaction in the Smoluchowski model. In $^{26}$ it is chosen to try to recover the stationary distribution of the corresponding non-spatial, well-mixed system. These procedures only work for sufficiently large lattice spacings ${ }^{34}$, for example a factor of $\pi$ times the reaction-radius in three-dimensions for the method of ${ }^{34}$.

The approach of ${ }^{35}$ involves several modifications to the RDME. The authors begin by considering the reaction $\mathrm{A}+\mathrm{B} \leftrightarrows \mathrm{C}$ for a system in which there is one stationary A molecule located at the origin, and one B molecule that may diffuse within a concentric sphere about the A molecule. (The A molecule is assumed to be a sphere.) The corresponding radiallysymmetric Smoluchowski model for the separation of the B molecule from the A molecule is then discretized into the form of a master equation, where the B molecule hops on a radial mesh bounded by the surface of the A molecule and the outer domain boundary. From this discretization, the authors determine an analytic, lattice spacing dependent bimolecular reaction rate when the $\mathrm{B}$ molecule is in the mesh voxel bordering the A molecule. For a given mesh spacing, this rate is chosen so that the mean equilibration time in the lattice model is the same as in the Smoluchowski model ${ }^{35}$. The authors then adapt their reaction rates to allow both molecules to move on a Cartesian grid lattice. To improve the accuracy

of the RDME in approximating the Smoluchowski model, in ${ }^{35}$ reactions are allowed between molecules within nearest neighbor voxels along each coordinate direction. For example, in two dimensions this leads to a five-point reaction stencil. Within this stencil, bimolecular reactions are modeled as well-mixed, and occur with a fixed rate based on volume corrections to the rate derived for the spherically symmetric model.

While the method of ${ }^{35}$ makes use of reactions between molecules in different voxels it is 
important to note it is fundamentally different than the CRDME we derive in Section III. First, the method of ${ }^{35}$ is designed to accurately approximate one particular statistic of the Smoluchowski model, the mean equilibration time, for a large range of lattice spacings that are bigger than some multiple of the reaction radius. (The simulations $\mathrm{in}^{35}$ are restricted to lattice spacings greater than twice the reaction-radius.) In contrast, our method is designed so that the solution to the CRDME converges to the solution of the Doi model as the lattice spacing is taken to zero (and hence should be able to approximate any statistic of that model to arbitrary accuracy, for sufficiently small lattice spacings). The method of ${ }^{35}$ allows a molecule to react with other molecules in a fixed number of neighboring voxels as the lattice spacing is changed. The volume of the region about one molecule in which a reaction can occur will therefore approach zero as the lattice spacing approaches zero. As in the standard RDME, this may cause a loss of bimolecular reactions should one try to take the lattice spacing to zero (which is not the goal of ${ }^{35}$ ). The CRDME decouples the region in which two molecules may react from the lattice, as described in the introduction. For lattice spacings greater than the reaction-radius an A molecule can react with B molecules in any nearest-neighbor voxel (including diagonal neighbors). For lattice spacings smaller than the reaction radius, an $\mathrm{A}$ molecule can react with $\mathrm{B}$ molecules in any voxels for which the minimum separation between any point in the A molecule voxel and any point in the B molecule voxel is smaller than the reaction radius. As such, the number of voxels separating two voxels for which a reaction can occur increases as the lattice spacing is decreased. The effective volume in which a reaction between two molecules is allowed approaches that of the Doi model as the lattice spacing is decreased to zero. Moreover, the probability per unit time two molecules will react is a function of the separation of the voxels that contain them, and is not constant across all voxel pairs for which a reaction can occur (in contrast to ${ }^{35}$ ).

It should also be noted that the version of the Smoluchowski model used in ${ }^{34,35}$ only takes into account the physical size of molecules in the context of bimolecular reactions. Volume exclusion due to molecule size is not accounted for in the diffusive hopping rates (which are chosen to recover the Brownian motion of point particles). With this approximation, the Doi model should provide a similar level of physical accuracy to the Smoluchowski model. For example, it was shown in ${ }^{43}$ that if molecules react instantly upon reaching a fixed separation in the Smoluchowski model, as in the popular Brownian dynamics simulator Smoldyn ${ }^{28}$, then the solution to the Doi model converges to the solution of the Smoluchowski model 
as the probability per unit time for two molecules to react when separated by less than a reaction-radius is increased to infinity. It was also shown in ${ }^{41}$ that the standard statistics one might wish to match in the Smoluchowski model, such as specified geminate recombination probabilities, can be captured by an appropriate choice of reaction parameters in the Doi model.

\section{GENERAL DOI AND RDME MODELS}

We first illustrate how the bimolecular reaction $\mathrm{A}+\mathrm{B} \rightarrow \mathrm{C}$ would be described by the continuum Doi model and the standard lattice RDME model in $\mathbb{R}^{d}$. In the Doi model, bimolecular reactions are characterized by two parameters; the separation at which molecules may begin to react, $r_{\mathrm{b}}$, and the probability per unit time the molecules react when within this separation, $\lambda$. When a molecule of species $\mathrm{A}$ and a molecule of species $\mathrm{B}$ react we assume the $\mathrm{C}$ molecule they produce is placed midway between them. Note the important point that in each of the models molecules are modeled as points.

We now formulate the Doi model as an infinite coupled system of partial integral differential equations (PIDEs). Let $\boldsymbol{q}_{l}^{a} \in \mathbb{R}^{d}$ denote the position of the $l$ th molecule of species A when the total number of molecules of species A is $a$. The state vector of the species A molecules is then given by $\boldsymbol{q}^{a}=\left(\boldsymbol{q}_{1}^{a}, \ldots, \boldsymbol{q}_{a}^{a}\right) \in \mathbb{R}^{d a}$. Define $\boldsymbol{q}^{b}$ and $\boldsymbol{q}^{c}$ similarly. We denote by $f^{(a, b, c)}\left(\boldsymbol{q}^{a}, \boldsymbol{q}^{b}, \boldsymbol{q}^{c}, t\right)$ the probability density for there to be $a$ molecules of species A, $b$

molecules of species $\mathrm{B}$, and $c$ molecules of species $\mathrm{C}$ at time $t$ located at the positions $\boldsymbol{q}^{a}$, $\boldsymbol{q}^{b}$, and $\boldsymbol{q}^{c}$. Molecules of the same species are assumed indistinguishable. The evolution of $f^{(a, b, c)}$ is given by

$$
\frac{\partial f^{(a, b, c)}}{\partial t}\left(\boldsymbol{q}^{a}, \boldsymbol{q}^{b}, \boldsymbol{q}^{c}, t\right)=(L+R) f^{(a, b, c)}\left(\boldsymbol{q}^{a}, \boldsymbol{q}^{b}, \boldsymbol{q}^{c}, t\right) .
$$

Note, with the subsequent definitions of the operators $L$ and $R$ this will give a coupled system of PIDEs over all possible values of $(a, b, c)$. More general systems that allow unbounded production of certain species would result in an infinite number of coupled PIDEs. The diffusion operator, $L$, is defined by

$$
L f^{(a, b, c)}=\left[D^{\mathrm{A}} \sum_{l=1}^{a} \Delta_{l}^{a}+D^{\mathrm{B}} \sum_{m=1}^{b} \Delta_{m}^{b}+D^{\mathrm{C}} \sum_{n=1}^{c} \Delta_{n}^{c}\right] f^{(a, b, c)},
$$

where $\Delta_{l}^{a}$ denotes the Laplacian in the coordinate $\boldsymbol{q}_{l}^{a}$ and $D^{\mathrm{A}}$ the diffusion constant of species A. $D^{\mathrm{B}}, D^{\mathrm{C}}, \Delta_{m}^{b}$, and $\Delta_{n}^{c}$ are defined similarly. 
To define the reaction operator, $R$, we introduce notations for removing or adding a specific molecule to the state $\boldsymbol{q}^{a}$. Let

$$
\begin{aligned}
\boldsymbol{q}^{a} \backslash \boldsymbol{q}_{l}^{a} & =\left(\boldsymbol{q}_{1}^{a}, \ldots, \boldsymbol{q}_{l-1}^{a}, \boldsymbol{q}_{l+1}^{a}, \ldots, \boldsymbol{q}_{a}^{a}\right) \\
\boldsymbol{q}^{a} \cup \boldsymbol{q} & =\left(\boldsymbol{q}_{1}^{a}, \ldots, \boldsymbol{q}_{a}^{a}, \boldsymbol{q}\right)
\end{aligned}
$$

$\boldsymbol{q}^{a} \backslash \boldsymbol{q}$ will denote $\boldsymbol{q}^{a}$ with any one component with the value $\boldsymbol{q}$ removed. Denote by $\mathbb{1}_{\left[0, r_{\mathrm{b}}\right]}(r)$ the indicator function of the interval $\left[0, r_{\mathrm{b}}\right]$. The Doi reaction operator, $R$, is then

$$
\begin{aligned}
&\left(R f^{(a, b, c)}\right)\left(\boldsymbol{q}^{a}, \boldsymbol{q}^{b}, \boldsymbol{q}^{c}, t\right)=\lambda\left[\sum_{l=1}^{c}\right. \\
& \int_{R^{d}} \int_{R^{d}} \delta\left(\frac{\boldsymbol{q}+\boldsymbol{q}^{\prime}}{2}-\boldsymbol{q}_{l}^{c}\right) \mathbb{1}_{\left[0, r_{\mathrm{b}}\right]}\left(\left|\boldsymbol{q}-\boldsymbol{q}^{\prime}\right|\right) f^{(a+1, b+1, c-1)}\left(\boldsymbol{q}^{a} \cup \boldsymbol{q}, \boldsymbol{q}^{b} \cup \boldsymbol{q}^{\prime}, \boldsymbol{q}^{c} \backslash \boldsymbol{q}_{l}^{c}, t\right) d \boldsymbol{q} d \boldsymbol{q}^{\prime} \\
& \\
&\left.\quad-\sum_{l=1}^{a} \sum_{l^{\prime}=1}^{b} \mathbb{1}_{\left[0, r_{\mathrm{b}}\right]}\left(\left|\boldsymbol{q}_{l}^{a}-\boldsymbol{q}_{l^{\prime}}^{b}\right|\right) f^{(a, b, c)}\left(\boldsymbol{q}^{a}, \boldsymbol{q}^{b}, \boldsymbol{q}^{c}, t\right)\right] .
\end{aligned}
$$

Let $B_{l}^{c}=\left\{\boldsymbol{q} \in \mathbb{R}^{d}|| \boldsymbol{q}-\boldsymbol{q}_{l}^{c} \mid \leq r_{\mathrm{b}} / 2\right\}$ label the set of points a reactant could be at to produce a molecule of species $\mathrm{C}$ at $\boldsymbol{q}_{l}^{c}$. Then (3) simplifies to

$$
\begin{array}{r}
\left(R f^{(a, b, c)}\right)\left(\boldsymbol{q}^{a}, \boldsymbol{q}^{b}, \boldsymbol{q}^{c}, t\right)=\lambda\left[2^{d} \sum_{l=1}^{c} \int_{B_{l}^{C}} f^{(a+1, b+1, c-1)}\left(\boldsymbol{q}^{a} \cup \boldsymbol{q}, \boldsymbol{q}^{b} \cup\left(2 \boldsymbol{q}_{l}^{c}-\boldsymbol{q}\right), \boldsymbol{q}^{c} \backslash \boldsymbol{q}_{l}^{c}, t\right) d \boldsymbol{q}\right. \\
\left.-\sum_{l=1}^{a} \sum_{l^{\prime}=1}^{b} \mathbb{1}_{\left[0, r_{\mathrm{b}}\right]}\left(\left|\boldsymbol{q}_{l}^{a}-\boldsymbol{q}_{l^{\prime}}^{b}\right|\right) f^{(a, b, c)}\left(\boldsymbol{q}^{a}, \boldsymbol{q}^{b}, \boldsymbol{q}^{c}, t\right)\right]
\end{array}
$$

We now describe the RDME, in a form we derived in $^{24}$ that has the advantage of representing a chemical system's state in a similar manner to the Doi model. Using this representation allows for easier comparison of the RDME and Doi models. Partition $\mathbb{R}^{d}$ into a Cartesian lattice of voxels with width $h$ and hypervolume $h^{d}$. When in the same voxel, an A and B molecule may react with probability per unit time $k / h^{d}$. Here $k$ represents the macroscopic bimolecular reaction-rate constant of the reaction $\mathrm{A}+\mathrm{B} \rightarrow \mathrm{C}$, with units of hypervolume per unit time. Let $\boldsymbol{j}_{l}^{a} \in \mathbb{Z}^{d}$ denote the multi-index of the voxel centered at $h \boldsymbol{j}_{l}^{a}$ that contains the $l$ th molecule of species A when there are $a$ molecules of species A. The position of the molecule is assumed to be uniformly distributed (i.e. well-mixed) within this voxel. Let $\boldsymbol{j}^{a}=\left(\boldsymbol{j}_{1}^{a}, \ldots, \boldsymbol{j}_{a}^{a}\right)$ denote the state vector for the voxels containing the $a$ molecules of species A. Define $\boldsymbol{j}^{b}$ and $\boldsymbol{j}^{c}$ similarly, and let $F_{h}^{(a, b, c)}\left(\boldsymbol{j}^{a}, \boldsymbol{j}^{b}, \boldsymbol{j}^{c}, t\right)$ denote the probability that there are $(a, b, c)$ molecules of species $\mathrm{A}, \mathrm{B}$, and $\mathrm{C}$ at time $t$ in the voxels given by $\boldsymbol{j}^{a}, \boldsymbol{j}^{b}$, and $\boldsymbol{j}^{c}$. 
The RDME is then the coupled system of ODEs over all possible values for $a, b, c, \boldsymbol{j}^{a}, \boldsymbol{j}^{b}$, and $\boldsymbol{j}^{c}$,

$$
\frac{d F_{h}^{(a, b, c)}}{d t}\left(\boldsymbol{j}^{a}, \boldsymbol{j}^{b}, \boldsymbol{j}^{c}, t\right)=\left(L_{h}+R_{h}\right) F_{h}^{(a, b, c)}\left(\boldsymbol{j}^{a}, \boldsymbol{j}^{b}, \boldsymbol{j}^{c}, t\right),
$$

where $L_{h}$ is a discretized approximation to $L$ given by

$$
L_{h} F_{h}^{(a, b, c)}\left(\boldsymbol{j}^{a}, \boldsymbol{j}^{b}, \boldsymbol{j}^{c}, t\right)=\left(D^{\mathrm{A}} \Delta_{h}^{a}+D^{\mathrm{B}} \Delta_{h}^{b}+D^{\mathrm{C}} \Delta_{h}^{c}\right) F_{h}^{(a, b, c)}\left(\boldsymbol{j}^{a}, \boldsymbol{j}^{b}, \boldsymbol{j}^{c}, t\right) .
$$

Here $\Delta_{h}^{a}$ denotes the standard $d a$-dimensional discrete Laplacian acting in the $\boldsymbol{j}^{a}$ coordinate. We define the "standard" $d$-dimensional discrete Laplacian acting on a mesh function, $f(\boldsymbol{j})$ on $\mathbb{Z}^{d}$, by

$$
\Delta_{h} f(\boldsymbol{j})=\frac{1}{h^{2}} \sum_{k=1}^{d}\left[f\left(\boldsymbol{j}+\boldsymbol{e}_{k}\right)+f\left(\boldsymbol{j}-\boldsymbol{e}_{k}\right)-2 f(\boldsymbol{j})\right],
$$

where $\boldsymbol{e}_{k}$ denotes a unit vector along the $k$ th coordinate axis of $\mathbb{R}^{d}$.

The reaction operator, $R_{h}$, is given by

$$
\begin{array}{r}
\left(R_{h} F_{h}^{(a, b, c)}\right)\left(\boldsymbol{j}^{a}, \boldsymbol{j}^{b}, \boldsymbol{j}^{c}, t\right)=\frac{k}{h^{d}}\left[\sum_{l=1}^{c} F_{h}^{(a+1, b+1, c-1)}\left(\boldsymbol{j}^{a} \cup \boldsymbol{j}_{l}^{c}, \boldsymbol{j}^{b} \cup \boldsymbol{j}_{l}^{c}, \boldsymbol{j}^{c} \backslash \boldsymbol{j}_{l}^{c}, t\right)\right. \\
\left.\quad-\sum_{l=1}^{a} \sum_{m=1}^{b} \delta_{h}\left(\boldsymbol{j}_{l}^{a}-\boldsymbol{j}_{m}^{b}\right) F_{h}^{(a, b, c)}\left(\boldsymbol{j}^{a}, \boldsymbol{j}^{b}, \boldsymbol{j}^{c}, t\right)\right],
\end{array}
$$

where $\delta_{h}\left(\boldsymbol{j}_{l}^{a}-\boldsymbol{j}_{m}^{b}\right)$ denotes the Kronecker delta function equal to one when $\boldsymbol{j}_{l}^{a}=\boldsymbol{j}_{m}^{b}$ and zero otherwise. Note that these equations are a formal discrete approximation to the Doi model, where two molecules may now react when in the same voxel with rate $k / h^{d}$.

We have shown that the RDME (4) may be interpreted as a formal approximation to both Doi-like and Smoluchowski models ${ }^{22,24,47}$. We proved in ${ }^{22}$, and showed numerically in ${ }^{47}$, that the RDME loses bimolecular reactions as $h \rightarrow 0$. This was demonstrated rigorously for $d=3$, where the time for two molecules two react was shown to diverge like $h^{-1}$. A simple modification of the argument in $^{22}$ shows bimolecular reactions are lost for all $d>1$, with a divergence like $\ln (h)$ for $d=2$, and like $h^{-d+2}$ for $d>2$. More recently asymptotic expansions were used in $^{34}$ to show the mean reaction time becomes infinite with the preceding rates as $h \rightarrow 0$ (for $d=2$ or $d=3$ ). This loss of reaction occurs because molecules are modeled by points, and as $h \rightarrow 0$ each voxel of the mesh shrinks to a point. Since two molecules must be in the same voxel to react, and in two or more dimensions two points can not find each other by diffusion, bimolecular reactions will never occur. 
It should be noted that while the RDME loses bimolecular reactions in the limit that $h \rightarrow$ 0 , we have shown that the solution to the RDME, for fixed values of $h$, gives an asymptotic approximation for small $r_{\mathrm{b}}$ to the solution of the Smoluchowski model ${ }^{22,47}$. How accurate this approximation can be made is dependent on domain geometry and the parameters of the underlying chemical system ${ }^{47}$. In particular, $h$ must be chosen sufficiently large that reactions within a voxel can be approximated by a well-mixed reaction with rate $k / h^{d}$, while chosen sufficiently small that the diffusion of the molecules is well-approximated by a continuous time random walk on the mesh ${ }^{7,22}$.

In the next section we develop a new convergent RDME (CRDME) to overcome these limitations of the standard RDME (4).

\section{A CONVERGENT RDME (CRDME)}

To construct a convergent RDME (CRDME) we use a finite volume discretization of the Doi PIDEs (1). For brevity we illustrate our approach on a simplified version of (1) when there is only one molecule of A and one molecule of B in the system which may undergo the annihilation reaction $\mathrm{A}+\mathrm{B} \rightarrow \varnothing$. The approach we describe can be extended to general multi-particle systems as bimolecular reactions in the Doi model only involve multiple twoparticle interactions of the same form, see (1).

For now we work in $d$-dimensional free-space, $\mathbb{R}^{d}$ (for most biological models $d=2$ or $d=3$ ). Denote by $\boldsymbol{x} \in \mathbb{R}^{d}$ the position of the molecule of species A and by $\boldsymbol{y} \in \mathbb{R}^{d}$ the position of the molecule of species B. In the Doi model these molecules diffuse independently, and may react with probability per unit time $\lambda$ when within a separation $r_{\mathrm{b}}$. $\left(r_{\mathrm{b}}\right.$ is usually called the reaction-radius.) We let $\mathcal{R}=\left\{(\boldsymbol{x}, \boldsymbol{y})|| \boldsymbol{x}-\boldsymbol{y} \mid<r_{\mathrm{b}}\right\}$, and denote the indicator function of this set by $\mathbb{1}_{\mathcal{R}}(|\boldsymbol{x}-\boldsymbol{y}|)$. The diffusion constants of the two molecules will be given by $D^{\mathrm{A}}$ and $D^{\mathrm{B}}$ respectively.

Finally, we denote by $p(\boldsymbol{x}, \boldsymbol{y}, t)$ the probability density the two molecules have not reacted and are at the positions $\boldsymbol{x}$ and $\boldsymbol{y}$ at time $t$. Then (1) reduces to

$$
\frac{\partial p}{\partial t}(\boldsymbol{x}, \boldsymbol{y}, t)=\left(D^{\mathrm{A}} \Delta_{\boldsymbol{x}}+D^{\mathrm{B}} \Delta_{\boldsymbol{y}}\right) p(\boldsymbol{x}, \boldsymbol{y}, t)-\lambda \mathbb{1}_{\mathcal{R}}(|\boldsymbol{x}-\boldsymbol{y}|) p(\boldsymbol{x}, \boldsymbol{y}, t) .
$$

(Here we have dropped the equation for the state $a=0, b=0$.)

We now show how to construct a new type of RDME by discretization of this equation. Note, while (7) can be solved analytically by switching to the separation coordinate, $\boldsymbol{x}-\boldsymbol{y}$, 


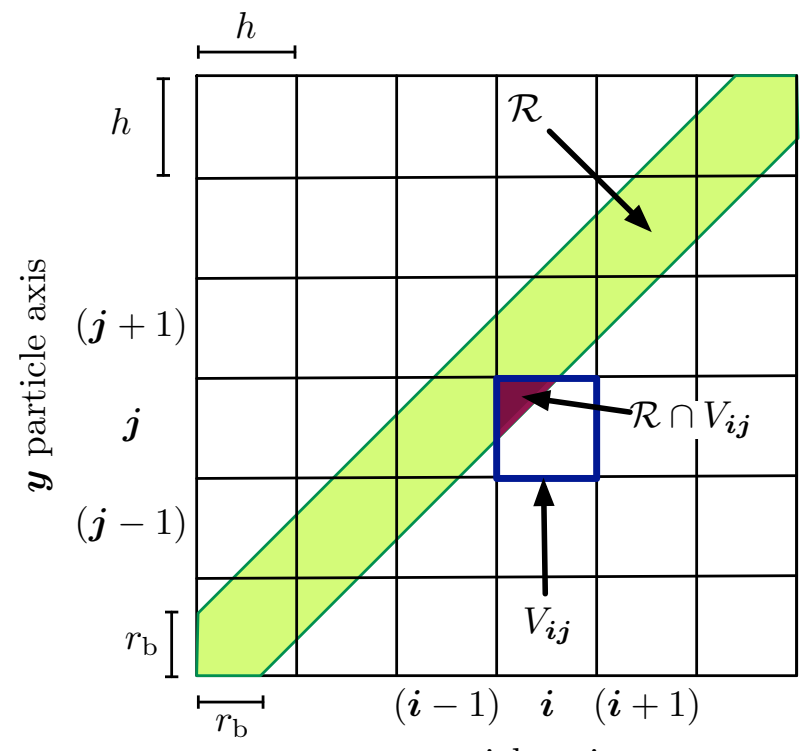

$\boldsymbol{x}$ particle axis

FIG. 1: Effective two-dimensional lattice when each particle is in $\mathbb{R}$. The green region corresponds to the set $\mathcal{R}$ of $(\boldsymbol{x}, \boldsymbol{y})$ values where the two molecules can react. $V_{\boldsymbol{i j}}$ labels the interior of the square with the blue boundary. It corresponds to the set of possible $(\boldsymbol{x}, \boldsymbol{y})$ values when the

A molecule with position $\boldsymbol{x}$ is randomly distributed within $\left[h\left(\boldsymbol{i}-\frac{1}{2}\right), h\left(\boldsymbol{i}+\frac{1}{2}\right)\right]$, and the B molecule with position $\boldsymbol{y}$ is randomly distributed within $\left[h\left(\boldsymbol{j}-\frac{1}{2}\right), h\left(\boldsymbol{j}+\frac{1}{2}\right)\right]$. The maroon region, $\mathcal{R} \cap V_{\boldsymbol{i j}}$, labels the subset of possible particle pair positions in $V_{\boldsymbol{i j}}$ where a reaction can occur.

such approaches will not work for more general chemical systems, such as (1). For this reason, we illustrate our CRDME ideas on (7). We later show in Section VI how the RDME reaction operator (6) for the general $\mathrm{A}+\mathrm{B} \rightarrow \mathrm{C}$ reaction is modified in the CRDME (see (13)).

For $\boldsymbol{i} \in \mathbb{Z}^{d}$ and $\boldsymbol{j} \in \mathbb{Z}^{d}$ we partition $\mathbb{R}^{2 d}$ into a Cartesian grid of hypercubes, labeled by $V_{\boldsymbol{i j}}$. We assume $V_{\boldsymbol{i} \boldsymbol{j}}$ has coordinate-axis aligned edges with length $h$ and center $\left(\boldsymbol{x}_{\boldsymbol{i}}, \boldsymbol{y}_{\boldsymbol{j}}\right)=(\boldsymbol{i} h, \boldsymbol{j} h)$. Denote the hypervolume of a set, $S$, by $|S|$. For example, the hypervolume of the hypercube $V_{\boldsymbol{i j}}$ is $\left|V_{\boldsymbol{i} \boldsymbol{j}}\right|=h^{2 d}$. In Fig. 1 we illustrate the various geometric quantities we will need in discretizing the Doi model (7) when the molecules are in one-dimension $(d=1)$.

We make the standard finite volume method approximation ${ }^{51}$ that the probability density, $p(\boldsymbol{x}, \boldsymbol{y}, t)$, is constant within each hypercube, $V_{\boldsymbol{i j}}$. The probability the two molecules are in $V_{\boldsymbol{i} \boldsymbol{j}}$ at time $t$ is then given by $P_{\boldsymbol{i}, \boldsymbol{j}}(t)=p(\boldsymbol{x}, \boldsymbol{y}, t)\left|V_{\boldsymbol{i} \boldsymbol{j}}\right|$ (for any point $(\boldsymbol{x}, \boldsymbol{y}) \in V_{\boldsymbol{i j}}$ ). Let $V_{\boldsymbol{i}}$ denote the $d$-dimensional hypercube with sides of length $h$ centered at $\boldsymbol{i} h$. With this 
definition we may write $V_{\boldsymbol{i} \boldsymbol{j}}=V_{\boldsymbol{i}} \times V_{\boldsymbol{j}} . P_{\boldsymbol{i}, \boldsymbol{j}}(t)$ then gives the probability that the particle of species $\mathrm{A}$ is in $V_{\boldsymbol{i}}$, and the particle of species B is in $V_{\boldsymbol{j}}$, at time $t$. The approximation that $p(\boldsymbol{x}, \boldsymbol{y}, t)$ is constant within $V_{\boldsymbol{i j}}$ is equivalent to assuming the two molecules are well-mixed within $V_{\boldsymbol{i}}$ and $V_{\boldsymbol{j}}$ respectively.

Using this assumption we construct a finite volume discretization of (7) by integrating both sides of $(7)$ over the hypercube $V_{\boldsymbol{i j}}$. As we did $\mathrm{in}^{21}$, we make the standard finite volume approximations for the integrals involving $\Delta_{x} p$ and $\Delta_{y} p$ to obtain discrete Laplacians given by (5) in the $\boldsymbol{x}$ and $\boldsymbol{y}$ coordinates. The reaction term is approximated by

$$
\begin{aligned}
\lambda \int_{V_{\boldsymbol{i} \boldsymbol{j}}} \mathbb{1}_{\mathcal{R}}(|\boldsymbol{x}-\boldsymbol{y}|) p(\boldsymbol{x}, \boldsymbol{y}, t) d \boldsymbol{x} d \boldsymbol{y} & \approx \frac{\lambda}{\left|V_{\boldsymbol{i} \boldsymbol{j}}\right|} P_{\boldsymbol{i}, \boldsymbol{j}}(t) \int_{V_{\boldsymbol{i} \boldsymbol{j}}} \mathbb{1}_{\mathcal{R}}(|\boldsymbol{x}-\boldsymbol{y}|) d \boldsymbol{x} d \boldsymbol{y} \\
& =\frac{\lambda\left|\mathcal{R} \cap V_{\boldsymbol{i j}}\right|}{\left|V_{\boldsymbol{i} \boldsymbol{j}}\right|} P_{\boldsymbol{i}, \boldsymbol{j}}(t) .
\end{aligned}
$$

Let $\phi_{\boldsymbol{i} \boldsymbol{j}}=\left|\mathcal{R} \cap V_{\boldsymbol{i j}}\right|\left|V_{\boldsymbol{i j}}\right|^{-1}$ label the fraction of the total volume in $V_{\boldsymbol{i j}}$ where a bimolecular reaction is possible (the maroon region in Fig. 1). $\phi_{\boldsymbol{i j}}$ is the probability that when the A molecule is well-mixed in $V_{\boldsymbol{i}}$ and the B molecule is well-mixed in $V_{\boldsymbol{j}}$ they are close enough to be able to react. Our discretization then represents a new RDME for the two-particle system, given by the coupled system of ODEs over all values of $(\boldsymbol{i}, \boldsymbol{j}) \in \mathbb{Z}^{2 d}$,

$$
\frac{d P_{\boldsymbol{i}, \boldsymbol{j}}}{d t}(t)=L_{h} P_{\boldsymbol{i}, \boldsymbol{j}}(t)-\lambda \phi_{\boldsymbol{i j}} P_{\boldsymbol{i}, \boldsymbol{j}}(t)
$$

Here $L_{h}=\left(D^{\mathrm{A}} L_{h}^{\mathrm{A}}+D^{\mathrm{B}} L_{h}^{\mathrm{B}}\right)$, with $L_{h}^{\mathrm{A}}$ and $L_{h}^{\mathrm{B}}$ denoting standard $d$-dimensional discrete Laplacians (5) (in the $\boldsymbol{i}$ and $\boldsymbol{j}$ coordinates respectively). By choosing an appropriate discretization we have obtained an equation that has the form of the forward Kolomogorov equation for a continuous-time jump Markov process. We may therefore interpret the coefficients in (8) as transition rates, also called propensities, between states $(\boldsymbol{i}, \boldsymbol{j})$ of the stochastic process. We subsequently refer to this equation as the CRDME. In the CRDME diffusion is handled in exactly the same manner as for the RDME. In contrast, the reaction mechanism in (8) now allows molecules in distinct voxels, $V_{\boldsymbol{i}}$ and $V_{\boldsymbol{j}}$, to react with a potentially non-zero probability per unit time, $\lambda \phi_{\boldsymbol{i j}}$.

As discussed earlier, the RDME is only physically valid when $h$ is chosen sufficiently large that the timescale for two molecules to become uniformly distributed within a voxel by diffusion is much faster than that for a well-mixed bimolecular reaction to occur between them. By allowing molecules to react when in nearby voxels, our CRDME provides a 
correction when $h$ is sufficiently small that this condition is violated. For $r_{\mathrm{b}}>h$ molecules may potentially react when separated by multiple voxels, with the number of voxels apart two molecules can be and still react increasing as $h \rightarrow 0$.

\section{NUMERICAL CONVERGENCE OF THE CRDME}

We now demonstrate that in two-dimensions $(d=2)$ the survival time distribution and the mean reaction time for the CRDME (8) converge to finite values as $h \rightarrow 0$. We show that in the corresponding RDME model the survival time and mean reaction time diverge to $\infty$ as $h \rightarrow 0$. In contrast, in the opposite limit that $r_{\mathrm{b}} / h \rightarrow 0$ we demonstrate that the mean reaction time in the RDME approaches that of the CRDME. As (7) is a PDE in four-dimensions, we do not directly solve the corresponding system of ODEs given by the CRDME (8). Instead, we simulate the corresponding stochastic jump process for the molecule's motion and reaction by the well-known exact stochastic simulation algorithm (SSA) (also known as the Gillespie method $^{31}$ or kinetic Monte Carlo $^{32}$ ).

We assume each molecule moves within a square with sides of length $L, \Omega=[0, L] \times[0, L]$, with zero Neumann boundary conditions. These boundary conditions are enforced by setting the transition rate for a molecule to hop from a given mesh voxel outside the domain to zero. For a specified number of mesh voxels, $N$, we discretize $\Omega$ into a Cartesian grid of squares with sides of length $h=L / N$. We assume $D^{\mathrm{A}}=D^{\mathrm{B}}=D$. Unless otherwise specified, all spatial units will be micrometers, with time in units of seconds. The SSA-based simulation algorithm can be summarized as:

1. Specify $D, L, N, \lambda$, and $r_{\mathrm{b}}$ as input.

2. Calculate $\phi_{\mathbf{0} \boldsymbol{j}}$. (See Appendix A.)

3. We assume the molecules are well-mixed at time $t=0$. That is, the initial position of each molecule is sampled from a uniform distribution among all voxels of the mesh.

4. Sample a time and direction of the next spatial hop by one of the molecules. (From (8) each molecule may hop to a neighbor in the $x$ or $y$ direction with probability per unit time $D / h^{2}$.)

5. Assuming the molecules are at $\boldsymbol{i}$ and $\boldsymbol{j}$, if $\phi_{\boldsymbol{i} \boldsymbol{j}} \neq 0$ sample the next reaction time using the transition rate $\lambda \phi_{i j}$. 
RDME

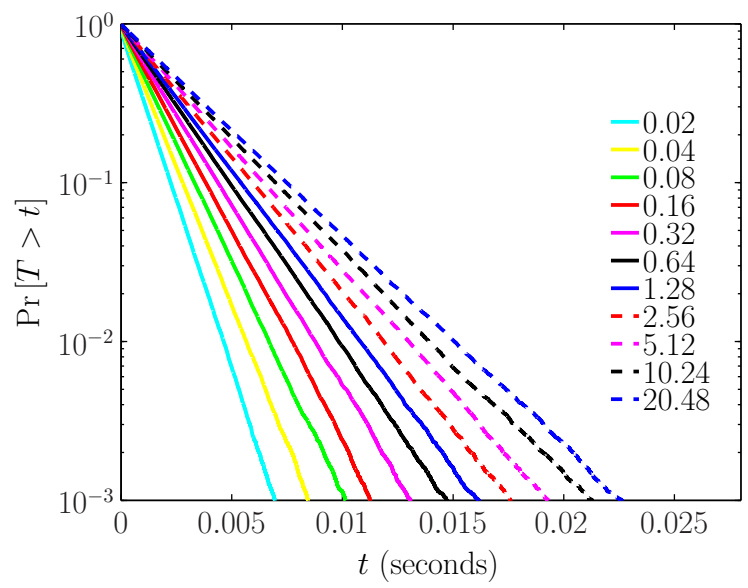

CRDME

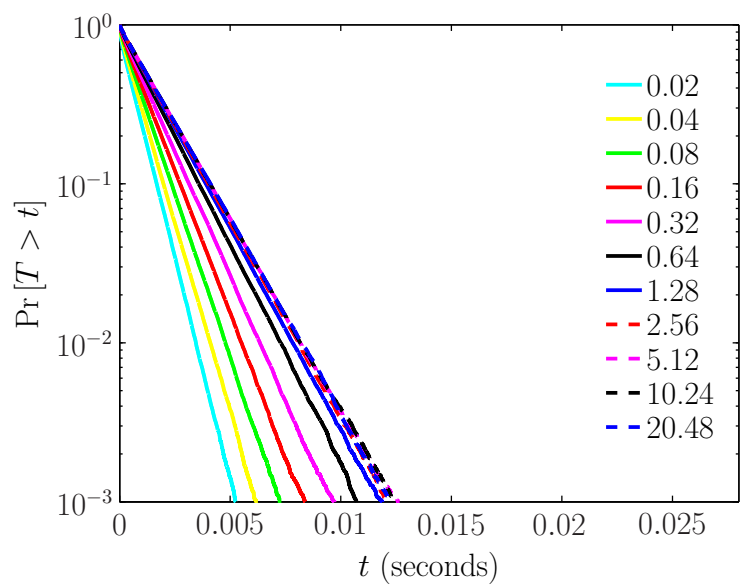

FIG. 2: Survival time distributions vs. $t$ for the CRDME and RDME when $\lambda=10^{9} \mathrm{~s}^{-1}$. Each curve was estimated from 128000 simulations. The legends give the ratio, $r_{\mathrm{b}} / h$, used for each curve (note that $r_{\mathrm{b}}=1 \mathrm{~nm}$ was fixed and $h$ successively halved). We see the convergence of the survival time distributions for the CRDME (up to sampling error), while the survival time distributions in the RDME diverge.

6. Select the smaller of the hopping and reaction times, and execute that event. Update the current time to the time of the event.

7. If a reaction occurs the simulation ends. If a spatial hop occurs, return to 4 .

For all simulations we chose $L=.2 \mu \mathrm{m}$. With this choice the domain could be interpreted as small patch of membrane within a cell. A diffusion constant of $D=10 \mu \mathrm{m}^{2} \mathrm{~s}^{-1}$ was used for each molecule. The reaction radius, $r_{\mathrm{b}}$, was chosen to be $1 \mathrm{~nm}$. While physical reaction radii are generally not measured experimentally, this choice falls between the measured width of the LexA DNA binding potential $\left(\approx 5\right.$ angstroms $\left.^{52}\right)$ and the $5 \mathrm{~nm}$ reaction radius used for interacting membrane proteins in $^{53}$.

We also simulated the stochastic process described by the corresponding RDME model. The bimolecular reaction rate was chosen to be $k=\lambda \pi r_{\mathrm{b}}^{2}$ to illustrate how the RDME approximates the CRDME as $r_{\mathrm{b}} / h \rightarrow 0$, but diverges as $r_{\mathrm{b}} / h \rightarrow \infty$. Our motivation for this choice is explained in the next section. The simulation algorithm was identical to that just described, except that step 2 was removed and step 5 modified so that two molecules could only react when within the same voxel (with probability per unit time $k=\lambda \pi r_{\mathrm{b}}^{2} / h^{2}$ ). 


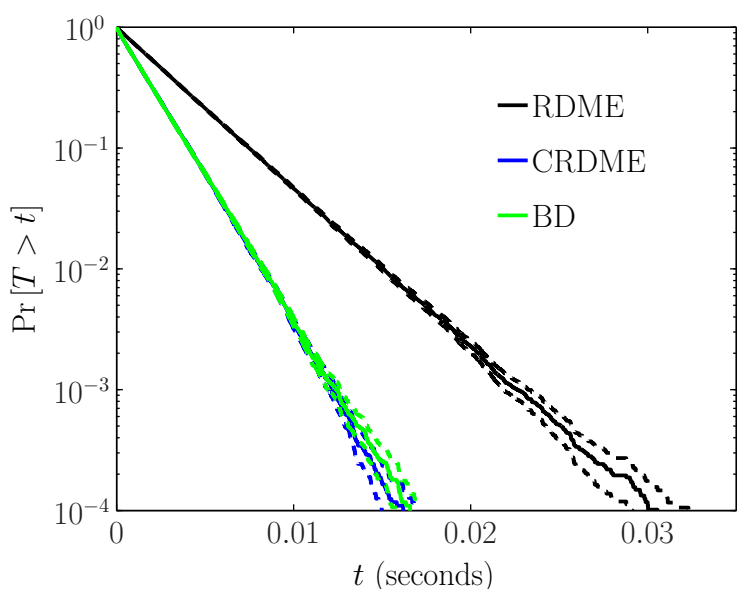

FIG. 3: Survival time distributions vs. $t$ for the RDME, CRDME, and from Brownian dynamics simulations (BD) when $\lambda=10^{9} \mathrm{~s}^{-1}$. The RDME and CRDME curves correspond to the survival time distributions shown in Fig. 2 for the largest value of $r_{\mathrm{b}} / h$. For each curve $95 \%$ confidence intervals are drawn with dashed lines (in the same color as the corresponding survival time distribution). They were determined using the Matlab ecdf routine. Each curve was estimated

from 128000 simulations. To statistical error the CRDME and BD simulations agree, demonstrating that the CRDME has recovered the survival time distribution of the Doi model.

Let $T$ denote the random variable for the time at which the two molecules react. The survival time distribution, $\operatorname{Pr}[T>t]$, is

$$
\operatorname{Pr}[T>t]=\int_{0}^{L} \int_{0}^{L} p(\boldsymbol{x}, \boldsymbol{y}, t) d \boldsymbol{x} d \boldsymbol{y} .
$$

Note that the reaction time distribution, $\operatorname{Pr}[T<t]=1-\operatorname{Pr}[T>t]$. We estimate $\operatorname{Pr}[T>t]$ from the numerically sampled reaction times using the MATLAB ecdf function. In Fig. 2 we show the convergence (to within sampling error) of the estimated survival time distributions for the CRDME (right figure) as $h \rightarrow 0$ (for $\lambda=10^{9} \mathrm{~s}^{-1}$ ). In the left figure we show the divergence as $h \rightarrow 0$ of the estimated survival time distributions in the RDME. The continuing rightward shift of the distribution as the mesh width is decreased to twenty times finer than the reaction radius shows the divergence of the reaction time to infinity.

To confirm that the CRDME was converging to the solution of the Doi model we repeated these studies for $\lambda=10^{9} \mathrm{~s}^{-1}$ using the Brownian dynamics (BD) method of ${ }^{26,41}$. In contrast to the RDME and CRDME, BD methods approximate the stochastic process describing the Brownian motion and reaction of the two molecules by discretization in time (instead of in 

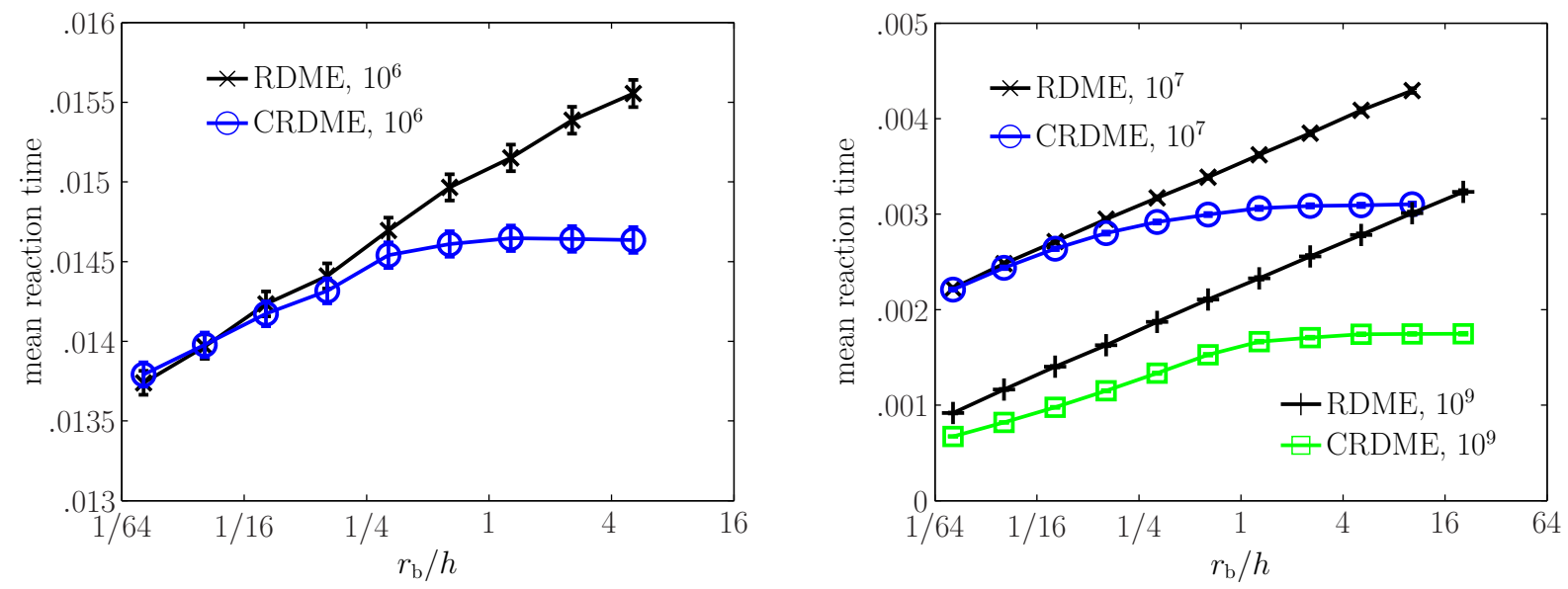

FIG. 4: Mean reaction times vs. $r_{\mathrm{b}} / h$ as $h$ decreases by factors of two. Legends give the value of $\lambda$ for each curve (with units of $\mathrm{s}^{-1}$ ). Each mean reaction time was estimated from 128000 simulations. Note that $95 \%$ confidence intervals are drawn on each data point. (For some points they are smaller than the marker labeling the point.) Since we use a logarithmic $x$-axis, we see that for $h$ sufficiently small the mean reaction time in the standard RDME diverges like $\ln (h)$, while in the CRDME the mean reaction time converges to a finite value.

space). For all BD simulations we used a fixed timestep, $d t=10^{-10} \mathrm{~s}$. We refer the reader to $^{41}$ for details of the specific BD method we used. Fig. 3 illustrates that the survival time distribution in the CRDME and BD method agree to statistical error (using the CRDME with the smallest value of $h$ from Fig 2). The CRDME therefore recovers the reaction time statistics of the Doi model as $h \rightarrow 0$.

The mean reaction time, $\mathbb{E}[T]$, is given by

$$
\mathbb{E}[T]=\int_{0}^{\infty} \operatorname{Pr}[T>t] d t .
$$

We estimated $\mathbb{E}[T]$ from the numerically sampled reaction times by calculating the sample mean. Fig. 4 shows the estimated mean reaction times for the CRDME and RDME models as $\lambda$ and $r_{\mathrm{b}} / h$ are varied (note the $x$-axis is logarithmic in $r_{\mathrm{b}} / h$ ). We see that as $h \rightarrow 0$ the sampled mean reaction times in the CRDME converge to a fixed value. For $\lambda=10^{9} \mathrm{~s}^{-1}$, the mean reaction time in the CRDME with the finest $h$ value, $0.0017471 \mathrm{~s}$, agreed with that found from the BD simulations used in Fig 3, 0.0017481s, to statistical error (i.e. within $95 \%$ confidence intervals, slightly less than $\pm 10^{-5}$ s about each mean value). The rate of convergence in the CRDME for $\lambda=10^{9}$ is illustrated in Fig. 5. There we plot the difference 


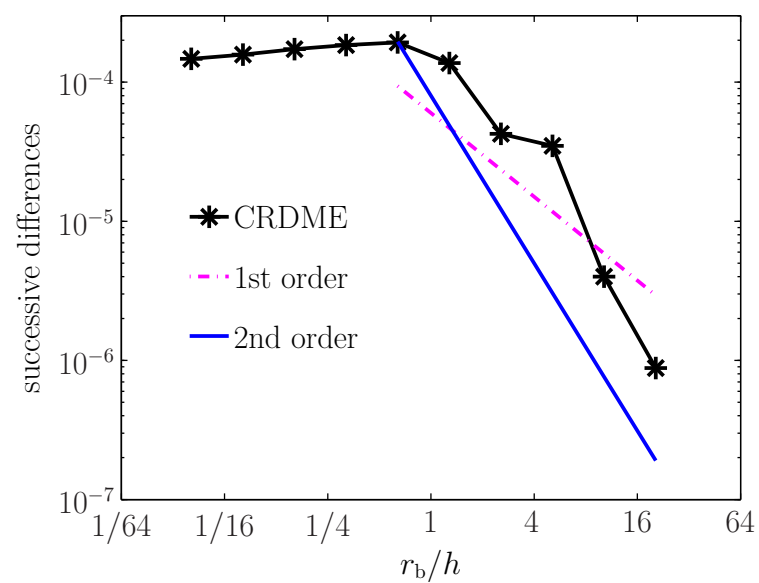

FIG. 5: Difference between successive points on the $\lambda=10^{9}$ CRDME curve in Fig. 4 vs. $r_{\mathrm{b}} / h$. The smaller of the two $h$ values is used to label each point. The first and second order curves scale like $h$ and $h^{2}$ respectively. Observe that the effective convergence rate to zero is closer to $O\left(h^{2}\right)$ than $O(h)$.

between successive estimated mean reaction times as $h$ is halved. For small values of $h$ this difference is seen to converge close to second order (as illustrated by the slope of the solid blue line).

In contrast, Fig. 4 shows that the sampled mean reaction time in the RDME diverges like $\ln (h)$ as discussed in ${ }^{22,34}$. For all $\lambda$ values the sampled mean reaction time in the RDME converges to that of the CRDME as $r_{\mathrm{b}} / h \rightarrow 0$. As $\lambda$ is decreased we see agreement between the RDME and CRDME for a larger range of $r_{\mathrm{b}} / h$ values.

Figs. 2, 4, and 5 demonstrate that, in contrast to the RDME, the reaction time statistics in the CRDME converge as $h \rightarrow 0$. For $\lambda=10^{9} \mathrm{~s}^{-1}$ we have verified the reaction time statistics of the CRDME recover those of the Doi model by comparison with BD simulations. In the large lattice limit that $r_{\mathrm{b}} / h \rightarrow 0$ we see that reaction time statistics of the RDME converge to those in the CRDME. Hence we may interpret the RDME as an approximation to the CRDME for $r_{\mathrm{b}} / h \ll 1$. The accuracy of using this approximation to describe the reactiondiffusion process in the Doi model will then depend on the relative sizes of $\lambda, D$, and $r_{\mathrm{b}}$ as we discuss in the next section (and illustrated in Fig. 4). 


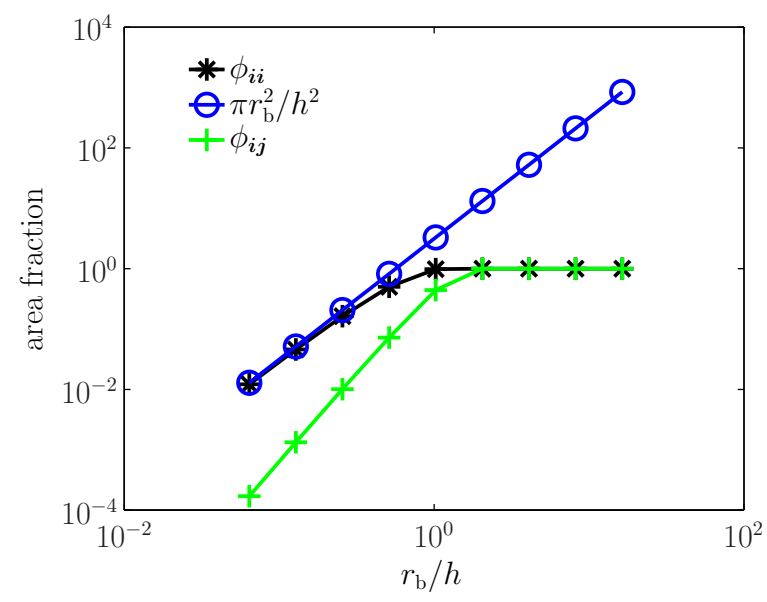

FIG. 6: Comparison of area fraction when both molecules are in the same square, $\phi_{\boldsymbol{i} i}$, with area fraction when the two molecules are in neighboring squares, $\phi_{\boldsymbol{i j}}$. We see that the area fraction decreases faster as $r_{\mathrm{b}} / h \rightarrow 0$ when the two molecules are in different squares than when they are in the same square. Moreover, as $r_{\mathrm{b}} / h \rightarrow 0$ the area fraction $\phi_{\boldsymbol{i} i}$ approaches $\pi r_{\mathrm{b}}^{2} / h^{2}$ as derived in (9).

\section{RDME AS AN APPROXIMATION OF THE CRDME FOR $r_{\mathbf{b}} / h \ll 1$}

We now show that the RDME may be interpreted as an asymptotic approximation to the CRDME for $r_{\mathrm{b}} / h \ll 1$, with the accuracy of this approximation depending on the size of $D, r_{\mathrm{b}}$, and $\lambda$. In the standard RDME two molecules can only react when within the same $d$-dimensional voxel. (If $k$ denotes a macroscopic bimolecular reaction rate, the probability per unit time the molecules react is usually chosen to be $k / h^{d}$, see (6).) In contrast, our new model allows two molecules to react when in neighboring voxels. Even for large values of $h$, the volume fraction $\phi_{\boldsymbol{i} j}$ will be non-zero when $\boldsymbol{i}$ and $\boldsymbol{j}$ are neighboring voxels. That said, for $\boldsymbol{j} \neq \boldsymbol{i}$ the volume fraction $\phi_{\boldsymbol{i} \boldsymbol{j}}$ will approach zero quicker as $r_{\mathrm{b}} / h \rightarrow 0$ than $\phi_{\boldsymbol{i} \boldsymbol{i}}$. This relationship is shown in two-dimensions $(d=2)$ in Fig. 6. (We describe how the area fractions were calculated in Appendix A.)

We therefore expect that, asymptotically, when $r_{\mathrm{b}} / h \rightarrow 0$ the particles will effectively 
only react when $\boldsymbol{j}=\boldsymbol{i}$. In this case

$$
\begin{aligned}
\left|\mathcal{R} \cap V_{\boldsymbol{i i}}\right| & =\int_{\mathcal{R} \cap V_{i i}} d \boldsymbol{y} d \boldsymbol{x} \\
& \approx \int_{[-h / 2, h / 2]^{d}} \int_{\left\{\boldsymbol{y}|| \boldsymbol{x}-\boldsymbol{y} \mid<r_{\mathrm{b}}\right\}} d \boldsymbol{y} d \boldsymbol{x} \\
& =\left|B_{r_{\mathrm{b}}}\right| h^{d},
\end{aligned}
$$

where $\left|B_{r_{\mathrm{b}}}\right|$ denotes the volume of the $d$-dimensional sphere of radius $r_{\mathrm{b}}$. The reaction rate when both particles are at the same position, $\boldsymbol{j}=\boldsymbol{i}$, is then

$$
\lambda \phi_{i \boldsymbol{i}}=\frac{\lambda\left|\mathcal{R} \cap V_{\boldsymbol{i i}}\right|}{\left|V_{\boldsymbol{i} i}\right|} \approx \frac{\lambda\left|B_{r_{\mathrm{b}}}\right|}{h^{d}} .
$$

This corresponds to the choice of bimolecular reaction rate $k=\lambda\left|B_{r_{\mathrm{b}}}\right|$ in the standard RDME. With this choice, when $r_{\mathrm{b}} / h \rightarrow 0$ the RDME may be interpreted as an asymptotic approximation of the CRDME. This approximation is illustrated in Fig. 4.

In three-dimensions, $d=3$, the macroscopic bimolecular reaction rate $k=\lambda\left|B_{r_{\mathrm{b}}}\right|$ also arises as the leading order asymptotic expansion as $r_{\mathrm{b}} \rightarrow 0, \lambda \rightarrow 0$, or $D=D^{\mathrm{A}}+D^{\mathrm{B}} \rightarrow \infty$ of the diffusion limited bimolecular reaction rate for the Doi model $(7), k_{\text {Doi }}$. $\operatorname{In}^{26}$ the latter was found to be

$$
k_{\text {Doi }}=4 \pi D r_{\mathrm{b}}\left(1-\frac{1}{r_{\mathrm{b}}} \sqrt{\frac{D}{\lambda}} \tanh \left(r_{\mathrm{b}} \sqrt{\frac{\lambda}{D}}\right)\right) .
$$

(Note, the more well-known Smoluchowski diffusion limited reaction rate $^{15,16}, k_{\text {Smol }}=$ $4 \pi D r_{\mathrm{b}}$, is recovered in the limit $\lambda \rightarrow \infty$.) As $r_{\mathrm{b}} \sqrt{\lambda / D} \rightarrow 0, k_{\text {Doi }} \sim \lambda\left(4 \pi r_{\mathrm{b}}^{3} / 3\right)=\lambda\left|B_{r_{\mathrm{b}}}\right|$. We thus have that the CRDME recovers this well-mixed reaction rate as $r_{\mathrm{b}} / h \rightarrow 0$. The smaller $r_{\mathrm{b}} \sqrt{\lambda / D}$, the better the RDME should approximate the CRDME for fixed $r_{\mathrm{b}} / h$.

When modeling three-dimensional biological systems, if $r_{\mathrm{b}} \sqrt{\lambda / D}$ is sufficiently small $h$ may simply be chosen to accurately model molecular diffusion by a continuous-time random walk. In this case, if $r_{\mathrm{b}} / h \ll 1$ we may approximate the CRDME by the standard RDME. When these assumptions break down we need to decrease $h$ and incorporate reactions between molecules in neighboring voxels with reactive transition rates $\lambda \phi_{\boldsymbol{i j}}$. The reaction and diffusion processes could potentially be decoupled by choosing separate meshes for each (as $\left.\mathrm{in}^{35}\right)$. That said, this process should be done so as to provide a convergent approximation of $(7)$. 


\section{REACTION PRODUCT PLACEMENT}

Consider again the bimolecular reaction, $\mathrm{A}+\mathrm{B} \rightarrow \mathrm{C}$. As previously described, the fundamental problem that prevents the standard RDME from converging to a reasonable continuous particle method is the inability for reactants to find each other as $h \rightarrow 0$. We therefore expect that any reasonable approach to placing a newly created $\mathrm{C}$ molecule will not impede the convergence of the CRDME. In this section we propose one possible scheme for use in the CRDME, consistent with the Doi model of Section II. In the Doi model a newly created $\mathrm{C}$ molecule was placed at the center of the line connecting the reacting $\mathrm{A}$ and B molecules. As molecules are assumed uniformly distributed within voxels in the CRDME, the method we now propose randomly places a newly created molecule in one of several possible voxels surrounding those containing two reactants.

To illustrate our approach we restrict to the two-particle $\mathrm{A}+\mathrm{B} \rightarrow \mathrm{C}$ reaction for molecules in $\mathbb{R}^{d}$. As in Section III we denote by $p(\boldsymbol{x}, \boldsymbol{y}, t)$ the probability density in the Doi model that the molecules of species A and B have not reacted and are located at $\boldsymbol{x}$ and $\boldsymbol{y}$ respectively at time $t$. $p(\boldsymbol{x}, \boldsymbol{y}, t)$ still satisfies (7), and will have the CRDME approximation (8). Let $u(\boldsymbol{q}, t)$ denote the probability density that a $\mathrm{C}$ molecule has been created and is located at position $\boldsymbol{q} \in \mathbb{R}^{d}$ at time $t$. If $D^{\mathrm{C}}$ denotes the diffusion constant of the $\mathrm{C}$ molecule then, similar to the in-flux term in the general Doi reaction operator $(3)$, we find $u(\boldsymbol{q}, t)$ satisfies

$$
\begin{aligned}
\frac{\partial u}{\partial t} & =D^{\mathrm{C}} \Delta_{\boldsymbol{q}} u+\lambda \int_{\mathbb{R}^{d}} \int_{\mathbb{R}^{d}} \delta\left(\frac{\boldsymbol{x}+\boldsymbol{y}}{2}-\boldsymbol{q}\right) \mathbb{1}_{\mathcal{R}}(|\boldsymbol{x}-\boldsymbol{y}|) p(\boldsymbol{x}, \boldsymbol{y}, t) d \boldsymbol{x} d \boldsymbol{y} \\
& =D^{\mathrm{C}} \Delta_{\boldsymbol{q}} u+2^{d} \lambda \int_{B_{\frac{r_{\mathbf{b}}}{2}}(\boldsymbol{q})} p(\boldsymbol{x}, 2 \boldsymbol{q}-\boldsymbol{x}, t) d \boldsymbol{x} .
\end{aligned}
$$

Here $\Delta_{\boldsymbol{q}}$ denotes the Laplacian in $\boldsymbol{q}$, while the second term corresponds to the in-flux of probability density produced by the reaction of the A and B molecules. $B_{\frac{r_{\mathrm{b}}}{2}}(\boldsymbol{q})$ denotes the hypersphere of radius $r_{\mathrm{b}} / 2$ centered at $\boldsymbol{q}$.

We now derive a master equation approximation of (10), consistent with the CRDME approximation (8) of (7). Discretize $\mathbb{R}^{d}$ into a lattice of cubic voxels of length $h$ indexed by $\boldsymbol{k} \in \mathbb{Z}^{d}$. The $\boldsymbol{k}$ th voxel is labeled by $V_{\boldsymbol{k}}$, with $\boldsymbol{q}_{\boldsymbol{k}}=\boldsymbol{k} h$ denoting the center of the voxel. We make the approximation that the probability the $\mathrm{C}$ molecule exists and is located in the voxel $V_{\boldsymbol{k}}$ at time $t$ is given by $U_{\boldsymbol{k}}(t)=u\left(\boldsymbol{q}_{\boldsymbol{k}}, t\right)\left|V_{\boldsymbol{k}}\right|$. Using this assumption we construct a finite volume discretization of (10) by integrating both sides of (10) over $V_{\boldsymbol{k}}$. We use the same finite volume approximation of the Laplacian as before, obtaining a discretized 
Laplacian in the $\boldsymbol{q}$ coordinate as defined in (5). Denote this discrete Laplacian by $D^{\mathrm{C}} L_{h}^{\mathrm{C}}$. The integral of the incoming flux term in (10) is approximated by

$$
\begin{aligned}
\int_{V_{\boldsymbol{k}}} \int_{\mathbb{R}^{d}} \int_{\mathbb{R}^{d}} \delta\left(\frac{\boldsymbol{x}+\boldsymbol{y}}{2}-\boldsymbol{q}\right) \mathbb{1}_{\mathcal{R}} & (|\boldsymbol{x}-\boldsymbol{y}|) p(\boldsymbol{x}, \boldsymbol{y}, t) d \boldsymbol{x} d \boldsymbol{y} d \boldsymbol{q} \\
& =\int_{\mathbb{R}^{d}} \int_{\mathbb{R}^{d}} \mathbb{1}_{V_{k}}\left(\frac{\boldsymbol{x}+\boldsymbol{y}}{2}\right) \mathbb{1}_{\mathcal{R}}(|\boldsymbol{x}-\boldsymbol{y}|) p(\boldsymbol{x}, \boldsymbol{y}, t) d \boldsymbol{x} d \boldsymbol{y} \\
& \approx \sum_{\substack{\boldsymbol{i} \in \mathbb{Z}^{d} \\
\boldsymbol{j} \in \mathbb{Z}^{d}}} \gamma_{\boldsymbol{i j}}^{\boldsymbol{k}} \phi_{\boldsymbol{i j}} P_{\boldsymbol{i}, \boldsymbol{j}}(t)
\end{aligned}
$$

Here $\mathbb{1}_{V_{\boldsymbol{k}}}(\boldsymbol{q})$ denotes the indicator function of the set $V_{\boldsymbol{k}}$ and $P_{\boldsymbol{i}, \boldsymbol{j}}(t)$ denotes the solution to the CRDME (8). $\gamma_{\boldsymbol{i} \boldsymbol{j}}^{\boldsymbol{k}}$ is defined by

$$
\gamma_{\boldsymbol{i j}}^{\boldsymbol{k}}= \begin{cases}\frac{1}{\phi_{\boldsymbol{i} j}\left|V_{\boldsymbol{i} j}\right|} \int_{V_{\boldsymbol{i j}}} \mathbb{1}_{V_{k}}\left(\frac{\boldsymbol{x}+\boldsymbol{y}}{2}\right) \mathbb{1}_{\mathcal{R}}(|\boldsymbol{x}-\boldsymbol{y}|) d \boldsymbol{x} d \boldsymbol{y}, & \phi_{\boldsymbol{i j}} \neq 0, \\ 0, & \phi_{\boldsymbol{i j}}=0\end{cases}
$$

and gives the probability that when $\boldsymbol{x} \in V_{\boldsymbol{i}}$ and $\boldsymbol{y} \in V_{\boldsymbol{j}}$ react the resultant $\mathrm{C}$ molecule is placed in voxel $V_{\boldsymbol{k}}$. Note,

$$
\sum_{k \in \mathbb{Z}^{d}} \gamma_{i j}^{k}=1
$$

when $\phi_{\boldsymbol{i} \boldsymbol{j}} \neq 0$. In practice, it is possible to calculate $\gamma_{\boldsymbol{i} \boldsymbol{j}}^{\boldsymbol{k}}$ by modification of the algorithm given in Appendix A for calculating $\phi_{\boldsymbol{i} \boldsymbol{j}}$.

We therefore arrive at the following master equation approximation of (10)

$$
\frac{d U_{\boldsymbol{k}}}{d t}=D^{\mathrm{C}} L_{h}^{\mathrm{C}} U_{\boldsymbol{k}}+\lambda \sum_{\substack{\boldsymbol{i} \in \mathbb{Z}^{d} \\ \boldsymbol{j} \in \mathbb{Z}^{d}}} \gamma_{\boldsymbol{i} \boldsymbol{j}}^{\boldsymbol{k}} \phi_{\boldsymbol{i j}} P_{\boldsymbol{i}, \boldsymbol{j}}(t) .
$$

Using the CRDME model given by (8) and (12) we are then led to a CRDME approximation of the general Doi model (1). Let $\tilde{F}_{h}^{(a, b, c)}\left(\boldsymbol{j}^{a}, \boldsymbol{j}^{b}, \boldsymbol{j}^{c}, t\right)$ denote the solution to the general CRDME, analogous to the solution of the standard RDME, $F_{h}^{(a, b, c)}\left(\boldsymbol{j}^{a}, \boldsymbol{j}^{b}, \boldsymbol{j}^{c}, t\right)$. $\tilde{F}_{h}$ also satisfies (4), with the same diffusion operator, $L_{h}$, but with the modified reaction operator, $\tilde{R}_{h}$, given by

$$
\begin{array}{r}
\left(\tilde{R}_{h} \tilde{F}_{h}^{(a, b, c)}\right)\left(\boldsymbol{j}^{a}, \boldsymbol{j}^{b}, \boldsymbol{j}^{c}, t\right)=\lambda\left[\sum_{l=1}^{c} \sum_{\substack{\boldsymbol{i} \in \mathbb{Z}^{d} \\
\boldsymbol{j} \in \mathbb{Z}^{d}}} \gamma_{\boldsymbol{i j}}^{c} \phi_{\boldsymbol{i j}} \tilde{F}_{h}^{(a+1, b+1, c-1)}\left(\boldsymbol{j}^{a} \cup \boldsymbol{i}, \boldsymbol{j}^{b} \cup \boldsymbol{j}, \boldsymbol{j}^{c} \backslash \boldsymbol{j}_{l}^{c}, t\right)\right. \\
\left.-\sum_{l=1}^{a} \sum_{m=1}^{b} \phi_{\boldsymbol{j}_{l}^{a} \boldsymbol{j}_{m}^{b}} \tilde{F}_{h}^{(a, b, c)}\left(\boldsymbol{j}^{a}, \boldsymbol{j}^{b}, \boldsymbol{j}^{c}, t\right)\right] .
\end{array}
$$




\section{MODIFIED SSA FOR BIMOLECULAR REACTIONS BASED ON THE CRDME}

We conclude by summarizing how to modify the SSA to generate realizations of the CRDME. Let $a_{i}$ denote the current number of molecules of species A in voxel $\boldsymbol{i}$ in a simulation, with $b_{j}$ and $c_{\boldsymbol{k}}$ defined similarly. We are then lead to the following proposed modification of the SSA to handle the bimolecular reaction $\mathrm{A}+\mathrm{B} \rightarrow \mathrm{C}$ based on the CRDME

1. The probability per unit time a molecule of species A in voxel $\boldsymbol{i}$ reacts with a molecule of species B in voxel $\boldsymbol{j}$ is given by the propensity $\lambda \phi_{\boldsymbol{i}} a_{\boldsymbol{i}} b_{\boldsymbol{j}}$.

2. Should such a reaction occur, update the system state so that

(a) $a_{i} \leftarrow a_{i}-1$

(b) $b_{j} \leftarrow b_{j}-1$.

(c) Randomly chose a voxel $\boldsymbol{k}$ with probability $\gamma_{i j}^{k}$ and update $c_{\boldsymbol{k}} \leftarrow c_{\boldsymbol{k}}+1$.

3. Recalculate any reaction or event times that depend on $a_{\boldsymbol{i}}, b_{\boldsymbol{j}}$, or $c_{\boldsymbol{k}}$.

For all diffusive transitions, zeroth order reactions, and first order reactions the SSA remains the same as for the standard $\mathrm{RDME}^{7}$.

\section{CONCLUSION}

By discretizing the stochastic reaction-diffusion model of $\operatorname{Doi}^{13,14}$ we have derived a new convergent reaction-diffusion master equation for $\mathrm{A}+\mathrm{B} \rightarrow \mathrm{C}$. We illustrated our discretization procedure, and the convergence of the survival time distribution and mean reaction time in the CRDME, for two molecules that undergo the annihilation reaction $\mathrm{A}+\mathrm{B} \rightarrow \varnothing$. While this special case is simplified compared to realistic biological networks, it should be noted that the same reaction rates, $\lambda \phi_{i j}$, are obtained by this discretization procedure for the more general multiparticle Doi model. This resulted in the general CRDME for $\mathrm{A}+\mathrm{B} \rightarrow \mathrm{C}$ given by (1) with the reaction operator (13). While we derived a convergent RDME by discretization of the Doi model in this work, we expect that a similar finitevolume discretization approach might also allow the derivation of a convergent RDME-like approximation to Smoluchowski models. 


\section{ACKNOWLEDGMENTS}

SAI is supported by NSF grant DMS-0920886. SAI thanks I. Agbanusi, D. Isaacson, and A. Steele for helpful comments and suggestions.

\section{Appendix A: Calculation of reaction transition rates}

Denote by $V_{\boldsymbol{i}}$ the $d$-dimensional coordinate axis aligned hypercube with sides of length $h$ centered at $\boldsymbol{i} h$. With this definition we may then write $V_{\boldsymbol{i} \boldsymbol{j}}=V_{\boldsymbol{i}} \times V_{\boldsymbol{j}}$. We use $\hat{V}_{\boldsymbol{i}}$ to denote this hypercube in the special case that $h=1$. Finally, let $B_{r_{\mathrm{b}}}(\boldsymbol{x})$ be the $d$-dimensional hypersphere of radius $r_{\mathrm{b}}$ about $\boldsymbol{x}$. A convenient representation for $\phi_{\boldsymbol{i}}$ we subsequently use is

$$
\begin{aligned}
\phi_{\boldsymbol{i j}} & =\frac{1}{\left|V_{\boldsymbol{i} \boldsymbol{j}}\right|} \int_{V_{\boldsymbol{i}}} \int_{V_{\boldsymbol{j}}} \mathbb{1}_{R}(|\boldsymbol{x}-\boldsymbol{y}|) d \boldsymbol{y} d \boldsymbol{x} \\
& =\frac{1}{\left|V_{\boldsymbol{i j}}\right|} \int_{V_{\boldsymbol{i}}}\left|B_{r_{\mathrm{b}}}(\boldsymbol{x}) \cap V_{\boldsymbol{j}}\right| d \boldsymbol{x} \\
& =\int_{\hat{V}_{\mathbf{0}}}\left|B_{\frac{r_{\mathrm{b}}}{h}}(\boldsymbol{x}) \cap \hat{V}_{\boldsymbol{j}-\boldsymbol{i}}\right| d \boldsymbol{x} .
\end{aligned}
$$

(Here $\mathbf{0}$ denotes the origin voxel.) Hence we may interpret $\phi_{\boldsymbol{i} \boldsymbol{j}}$ as the integral over the center of a hypersphere of the volume of intersection between the hypersphere and a hypercube. The final equation (A2) shows that $\phi_{\boldsymbol{i} \boldsymbol{j}}$ depends on only two quantities; the separation vector $\boldsymbol{j}-\boldsymbol{i}$ and $r_{\mathrm{b}} / h$. Also note that $\phi_{\boldsymbol{i} \boldsymbol{j}}$ will be zero once the separation between all points in voxels $\boldsymbol{i}$ and $\boldsymbol{j}$ is more than $r_{\mathrm{b}}$. As such, in practice it is only necessary to calculate $\phi_{\mathbf{0} \boldsymbol{j}}$ for a small number of voxels about the origin.

It is desirable to calculate $\phi_{\boldsymbol{i j}}$ to near machine precision to avoid the introduction of error from the use of incorrect reactive transition rates. While this may seem an easy task, simply calculating the hypervolume of intersection of $\mathcal{R}$ and $V_{\boldsymbol{i j}}$, it should be noted that these are four-dimensional (six-dimensional) sets when the molecules are in two-dimensions (threedimensions). Evaluating $\phi_{\boldsymbol{i} \boldsymbol{j}}$ by directly applying quadrature to (A1) is complicated by the discontinuous integrand. We have found that several standard cubature ${ }^{54,55}$ and Monte Carlo methods ${ }^{54}$ have difficultly evaluating such integrals in reasonable amounts of computing time to high numerical precision (absolute errors below $10^{-11}$ ). Since the integral (A2) has a continuous integrand, which only requires the intersection of two-dimensional (three- 
dimensional) sets when the particles are each in two-dimensions (three-dimensions), we focus on evaluating $\phi_{i j}$ through this representation.

To evaluate (A2) both efficiently and accurately it is necessary to calculate the hypervolume of intersection given by the integrand, $v_{\boldsymbol{j}}(\boldsymbol{x})=\left|B_{\frac{r_{\mathrm{b}}}{h}}(\boldsymbol{x}) \cap \hat{V}_{\boldsymbol{j}}\right|$. Our approach is based on writing this hypervolume as an integral and then converting to a boundary integral through the use of the divergence theorem. That is,

$$
\begin{aligned}
v_{\boldsymbol{j}}(\boldsymbol{x}) & =\frac{1}{d} \int_{B_{\frac{r_{\mathrm{b}}}{h}}(\boldsymbol{x}) \cap \hat{V}_{\boldsymbol{j}}} \nabla \cdot \boldsymbol{y} d \boldsymbol{y} \\
& =\frac{1}{d} \int_{\partial\left(B_{\frac{r_{b}}{h}}(\boldsymbol{x}) \cap \hat{V}_{\boldsymbol{j}}\right)} \boldsymbol{y} \cdot \boldsymbol{\eta}(\boldsymbol{y}) d S(\boldsymbol{y}), \\
& =\frac{1}{d} \int_{\partial B_{\frac{r_{\mathrm{b}}}{h}}(\boldsymbol{x})}(\boldsymbol{y} \cdot \boldsymbol{\eta}(\boldsymbol{y})) \mathbb{1}_{\hat{V}_{j}}(\boldsymbol{y}) d S(\boldsymbol{y})+\frac{1}{d} \int_{\partial \hat{V}_{j}}(\boldsymbol{y} \cdot \boldsymbol{\eta}(\boldsymbol{y})) \mathbb{1}_{B_{\frac{r_{\mathrm{b}}}{h}}(\boldsymbol{x})}(\boldsymbol{y}) d S(\boldsymbol{y}) .
\end{aligned}
$$

Here $\partial M$ is used to denote the boundary of a manifold $M, \boldsymbol{\eta}(\boldsymbol{y})$ the outward normal to the boundary hypersurface at $\boldsymbol{y}$, and $d S(\boldsymbol{y})$ the hypersurface measure at $\boldsymbol{y}$.

For simplicity, in the remainder we assume $d=2$. In this case we have developed a fast method, requiring only a few minutes on a modern laptop, that is able to evaluate (A2) to near machine precision. $v_{\boldsymbol{j}}(\boldsymbol{x})$ is evaluated by calculating the intersection points of the

circle $\partial B_{r_{\mathrm{b}}}(\boldsymbol{x})$ with the square $\hat{V}_{\boldsymbol{j}}$ numerically. Once these points are known the line integrals in (A3) can be reduced to sums of integrals over sub-arcs where the indicator function is identically one or zero. These integrals can be evaluated analytically. Standard adaptive numerical quadrature methods, such as the dblquad routine in MATLAB, are then able to effectively integrate the area of intersection function $v_{\boldsymbol{j}}(\boldsymbol{x})$. This method was used to generate the area fractions in Fig. 6 and all SSA simulations.

\section{REFERENCES}

${ }^{1}$ S. Neves, P. Tsokas, A. Sarkar, E. Grace, P. Rangamani, S. Taubenfeld, C. Alberini, J. Schaff, R. Blitzer, I. Moraru, and R. Iyengar, Cell 133, 666 (2008).

${ }^{2}$ B. Hendriks, L. Opresko, H. Wiley, and D. Lauffenburger, J. Biol. Chem. 278, 23343 (2003).

${ }^{3}$ G. Von Dassow, E. Meir, E. M. Munro, and G. M. Odell, Nature 406, 188 (2000).

${ }^{4}$ L. Ma, J. Wagner, J. J. Rice, W. Hu, A. J. Levine, and G. A. Stolovitzky, Proc. Natl. Acad. Sci. USA 102, 14266 (2005). 
${ }^{5}$ J. Muñoz-García, Z. Neufeld, B. N. Kholodenko, and H. M. Sauro, PLoS Comp. Biol. 5, e1000330 (2009).

${ }^{6}$ S. A. Isaacson, D. M. McQueen, and C. S. Peskin, PNAS 108, 3815 (2011).

${ }^{7}$ J. Elf and M. Ehrenberg, IEE Sys. Biol. 1, 230 (2004).

${ }^{8}$ A. Arkin and H. H. McAdams, Proc. Natl. Acad. Sci. USA 94, 814 (1997).

${ }^{9}$ W. J. Blake, M. Kaern, C. R. Cantor, and J. J. Collins, Nature 422, 633 (2003).

${ }^{10}$ J. Raser and E. O'Shea, Science 304, 1811 (2004).

${ }^{11}$ D. Shaw, R. Dror, J. Salmon, J. Grossman, K. Mackenzie, J. Bank, C. Young, M. Deneroff, B. Batson, and K. Bowers, Proceedings of the Conference on High Performance Computing Networking, Storage and Analysis , 39 (2009).

${ }^{12}$ E. Teramoto and N. Shigesada, Prog. Theor. Phys. 37, 29 (1967).

${ }^{13}$ M. Doi, J. Phys. A: Math. Gen. 9, 1465 (1976).

${ }^{14}$ M. Doi, J. Phys. A: Math. Gen. 9, 1479 (1976).

${ }^{15}$ M. V. Smoluchowski, Z. Phys. Chem. 92, 129 (1917).

${ }^{16}$ J. Keizer, J. Phys. Chem. 86, 5052 (1982).

${ }^{17}$ C. W. Gardiner, K. J. McNeil, D. F. Walls, and I. S. Matheson, J. Stat. Phys. 14, 307 (1976).

${ }^{18} \mathrm{C}$. W. Gardiner, Handbook of Stochastic Methods: For Physics, Chemistry, and the Natural Sciences, 2nd ed., Springer Series in Synergetics, Vol. 13 (Springer Verlag, New York, 1996).

${ }^{19}$ N. G. Van Kampen, Stochastic Processes in Physics and Chemistry (North-Holland, Amsterdam, 2001).

${ }^{20}$ R. Erban, S. J. Chapman, and P. K. Maini, "A practical guide to stochastic simulations of reaction-diffusion processes," (2007), arXiv:0704.1908 [q-bio.SC].

${ }^{21}$ S. A. Isaacson and C. S. Peskin, SIAM J. Sci. Comput. 28, 47 (2006).

${ }^{22}$ S. A. Isaacson, SIAM J. Appl. Math. 70, 77 (2009).

${ }^{23}$ D. A. McQuarrie, J. Appl. Prob. 4, 413 (1967).

${ }^{24}$ S. A. Isaacson, J. Phys. A: Math. Theor. 41, 065003 (15pp) (2008).

${ }^{25}$ S. Engblow, L. Ferm, A. Hellander, and P. Lötstedt, SIAM J. Sci. Comp. 31, 1774 (2009).

${ }^{26}$ R. Erban and S. J. Chapman, Phys. Biol. 6, 046001 (2009).

${ }^{27}$ R. A. Kerr, T. M. Bartol, B. Kaminsky, M. Dittrich, J. Chang, S. B. Baden, T. J. Sejnowski, and J. R. Stiles, SIAM J. Sci. Comput. 30, 3126 (2008).

${ }^{28}$ S. S. Andrews and D. Bray, Physical Biology 1, 137 (2004). 
${ }^{29}$ A. Donev, V. V. Bulatov, T. Oppelstrup, G. H. Gilmer, B. Sadigh, and M. H. Kalos, J. Comp. Phys. 229, 3214 (2010).

${ }^{30}$ K. Takahashi, S. Tanase-Nicola, and P. R. ten Wolde, PNAS 107, 2473 (2010).

${ }^{31}$ D. T. Gillespie, J. Phys. Chem. 81, 2340 (1977).

${ }^{32}$ A. B. Bortz, M. H. Kalos, and J. L. Lebowitz, J. Comp. Phys. 17, 10 (1975).

${ }^{33}$ S. Arjunan and M. Tomita, Syst Synt Biol 4, 35 (2010).

${ }^{34}$ S. Hellander, A. Hellander, and L. Petzold, Phys. Rev. E 85, 042901(1 (2012).

${ }^{35}$ D. Fange, O. G. Berg, P. Sjöberg, and J. Elf, PNAS 107, 19820 (2010).

${ }^{36}$ D. T. Gillespie, "Voxel size restrictions in the reaction-diffusion master equation," (2013), private communication.

${ }^{37}$ W. Naumann, J. Chem. Phys. 98, 2353 (1993).

${ }^{38}$ V. Kuzovkov and E. Kotomin, Reports on Progress in Physics 51, 1479 (1988).

${ }^{39}$ K. Seki, A. I. Shushin, M. Wojcik, and M. Tachiya, J. Phys.: Condens. Matter 19, 065117 (2007).

${ }^{40}$ M. Klann, A. Ganguly, and H. Koeppl, Bioinformatics 28, i549 (2012).

${ }^{41}$ J. Lipkova, K. C. Zygalakis, S. J. Chapman, and R. Erban, SIAM J. Appl. Math. 71, 714 (2011).

${ }^{42}$ M. B. Flegg, S. Rüdiger, and R. Erban, J. Chem. Phys. 138, 154103 (2013).

${ }^{43}$ I. C. Agbanusi and S. A. Isaacson, Bull. Math. Biol. (2013), 10.1007/s11538-013-9833-6.

${ }^{44}$ H. Wang, C. S. Peskin, and T. C. Elston, J. Theor. Biol. 221, 491 (2003).

${ }^{45}$ A. Hellander and P. Lötstedt, Multiscale Modeling \& Simulation 8, 1691 (2010).

${ }^{46}$ B. Bayati, P. Chatelain, and P. Koumoutsakos, J. Comp. Phys. 230, 13 (2011).

${ }^{47}$ S. A. Isaacson and D. Isaacson, Phys. Rev. E 80, 066106 (9pp) (2009).

${ }^{48}$ C. A. Yates, R. E. Baker, R. Erban, and P. K. Maini, Phys. Rev. E 86, 021921 (2012).

${ }^{49}$ M. Vigelius and B. Meyer, PloS one 7, e33384 (2012).

${ }^{50}$ L. Ferm, A. Hellander, and P. Lötstedt, J. Comp. Phys. 229, 343 (2010).

${ }^{51}$ Z. Cai, J. Douglas Jr, and M. Park, Adv. Comp. Math. 19, 3 (2003).

${ }^{52}$ F. Kühner, L. T. Costa, P. M. Bisch, S. Thalhammer, W. M. Heckl, and H. E. Gaub, Biophys. J. 87, 2683 (2004).

${ }^{53}$ O. Dushek, P. A. van der Merwe, and V. Shahrezaei, Biophys. J. 100, 1189 (2011).

${ }^{54}$ T. Hahn, Comp. Phys. Comm. 168, 78 (2005).

${ }^{55}$ S. G. Johnson, "Cubature integration library," Available at: 
http://ab-initio.mit.edu/wiki/index.php/Cubature. 